\title{
EURADOS IC2012N: EURADOS 2012 INTERCOMPARISON FOR WHOLE-BODY NEUTRON DOSIMETRY
}

\author{
E. Fantuzzi1,*, M.-A. Chevallier ${ }^{2}$, R. Cruz-Suarez ${ }^{3}$, M. Luszik-Bhadra ${ }^{4}$, S. Mayer ${ }^{5}$, D. J. Thomas ${ }^{6}$, R. Tanner $^{7}$ \\ and F. Vanhavere ${ }^{8}$ \\ ${ }^{1}$ ENEA-Radiation Protection Institute, via dei Colli, 16-40136 Bologna (BO), Italy \\ ${ }^{2}$ Institut de Radioprotection et de Sûreté Nucléaire (IRSN), BP 17, 31 avenue de la Division Leclerc, \\ 92260 Fontenay-aux-Roses, France \\ ${ }^{3}$ IAEA, UN-Wien, Austria \\ ${ }^{4}$ Physikalisch-Technische Bundesanstalt (PTB), Bundesallee 100, D-38116 Braunschweig, Germany \\ ${ }^{5}$ Paul Scherrer Institute (PSI), CH-5232 Villigen PSI, Switzerland \\ ${ }^{6}$ National Physical Laboratory (NPL), Hampton Road, Teddington TW11 0LW, UK \\ ${ }^{7}$ Public Health England, CRCE, Chilton, Didcot, Oxon OX11 0RQ, UK \\ ${ }^{8}$ SCK-CEN, Belgian Nuclear Research Centre, Boeretang 200, 2400 Mol, Belgium \\ *Corresponding author: elena.fantuzzi@enea.it
}

\begin{abstract}
The European Radiation Dosimetry Group (EURADOS) IC2012n intercomparison for neutron dosemeters intended to measure personal dose equivalent, $H_{\mathrm{p}}(\mathbf{1 0})$, was performed in 2012. A total of 31 participants $(27$ individual monitoring services from Europe, 2 from Japan, 1 from Israel and 1 from USA) registered with 34 dosimetry systems. Participation was restricted to passive or active neutron dosemeters routinely used in individual monitoring of radiation workers. The dosimetry systems were based on thermoluminescence, polyallyldiglycol carbonate, optically stimulated luminescence, fission track detection and silicon diodes (electronic devices). The irradiation tests were chosen to provide the participants with useful information on their dosimetry systems, i.e. linearity, reproducibility, responses for different energies and angles and to simulated workplace fields. The paper will report and discuss the first analysis of the results of the EURADOS IC2012n intercomparison.
\end{abstract}

\section{INTRODUCTION}

Although regular performance tests or intercomparisons are strongly advised in the recently updated European Commission's Technical Recommendations for Monitoring Individuals Occupationally Exposed to External Radiation ${ }^{(1)}$, they are carried out only in a few European countries. The European Radiation Dosimetry Group (EURADOS), as part of the work performed by its Working Group 2-Harmonization of Individual Monitoring in Europe, has started a self-sustained programme of regular intercomparisons ${ }^{(2)}$ and has successfully performed three intercomparisons for whole-body photon dosemeters (IC2008, IC2010, IC2012) and one intercomparison for extremity dosemeters for photon and beta fields (IC2009). In 2012, the EURADOS IC2012n intercomparison for neutron personal dosemeters designed to measure personal dose equivalent, $H_{\mathrm{p}}(10)$, was performed. It was meant for dosemeters provided by individual monitoring services (IMSs) for exposed workers. Systems under development were not allowed.

\section{THE EURADOS 2012 INTERCOMPARISON FOR WHOLE-BODY NEUTRON DOSIMETRY}

The aim of the intercomparison was to provide IMSs for external dosimetry with the opportunity to test their performance, to compare their results with other IMSs and to show compliance with their own management system. Participation was on a voluntary basis, given a participation fee.

The results are provided to the participants in the Certificate of Participation, with the certificates of the Calibration Laboratories, together with the signed copy of the results provided by the participants (prior to knowing the reference values) as annexes.

\section{Participants and dosimetry systems}

A total of 31 participants comprising 27 IMSs from 15 European countries, 2 from Japan, 1 from Israel and 1 from the USA, registered with 34 dosimetry systems. Each participant provided 36 dosemeters: 24 to be irradiated, 8 spare dosemeters and 4 background dosemeters.

According to the information provided by the participants, most of the dosimetry systems were albedo dosemeters based on thermoluminescence or etched track detectors, i.e. proton recoil dosemeters, based on polyallyl diglycol carbonate (PADC) or a combination of the above-mentioned detectors. In addition, two systems were based on optically stimulated luminescence (OSL), one was a fission track dosemeter and two were electronic devices based on silicon diodes. 


\section{E. FANTUZZI ET AL.}

Results are reported according to the following classification:

- Etched track: 18 systems (5 Track detectors for fast neutrons and TLD for albedo, 9 track detectors for fast neutrons combined with converters for thermal neutrons and 4 track detectors for fast neutrons only) (no evidence of a thermal sensor).

- Albedo: 13 systems (3 albedo TLD + Cd shield, 6 albedo TLD + boron loaded shield and 4 albedo TLD or OSL) (no information on shielding of direct thermal neutrons).

- Other: 3 systems (1 fission track and 2 electronic).

Complete results were received for 32 systems (30 passive, 2 active): 1 participant withdrew 1 system after registration, while another service was unable to provide useful results because of reader problems.

\section{Irradiations}

The IC2012n is not meant for mixed neutron-gamma fields but only to neutron dosimetry. Therefore, the irradiations have been restricted to neutrons: no additional photon irradiations were included over and above the photons associated with the neutron-production mechanism.

The irradiation tests were performed at two European accredited laboratories, which are both National Primary Metrology Laboratories for ionising radiation: NPL (National Physical Laboratory, UK) and PTB (Physikalisch-Technische Bundesanstalt, D).

The following irradiation plan was performed in a random order for each dosimetry system:

- $H_{\mathrm{p}}(10)=0.3,3$ and $15 \mathrm{mSv}$ with a bare ${ }^{252} \mathrm{Cf}$ source at $0^{\circ}$ (four dosemeters per dose value);

- $H_{\mathrm{p}}(10)=2 \mathrm{mSv}$ with a bare ${ }^{252} \mathrm{Cf}$ source at $45^{\circ}$ (two dosemeters);

- $H_{\mathrm{p}}(10)=3 \mathrm{mSv}$ with a $\left(\mathrm{D}_{2} \mathrm{O}\right)$ moderated ${ }^{252} \mathrm{Cf}$ source at $0^{\circ}$ (four dosemeters);

- $\quad H_{\mathrm{p}}(10)=2 \mathrm{mSv}$ with a bare ${ }^{252} \mathrm{Cf}$ source behind a shadow cone (two dosemeters);

- $H_{\mathrm{p}}(10)=1 \mathrm{mSv}$ with monoenergetic $250 \mathrm{keV}$ neutrons (four dosemeters) at $0^{\circ}$.

The irradiation tests were established by the organising group (OG) with the aim to test the dosimetry systems but also to provide the participants with calibration values for one International Organization for Standardisation (ISO) standard radionuclide source (i.e. ${ }^{252} \mathrm{Cf}$ source), and, for the same source, information on the performance of their dosimetry systems in terms of linearity (i.e. three dose values), reproducibility (i.e. four dosemeters for each irradiation condition), response to different angles (i.e. $0^{\circ}$ and $45^{\circ}$ ). Response to different energies was also tested adding irradiation tests with simulated workplace fields currently available at European accredited laboratories [i.e. $\left(\mathrm{D}_{2} \mathrm{O}\right)$ moderated ${ }^{252} \mathrm{Cf}$ source and bare ${ }^{252} \mathrm{Cf}$ source behind a shadow cone, that is energies from thermal to a few $\mathrm{MeV}$ ] and the monoenergetic 250$\mathrm{keV}$ neutron fields. The latter was also useful to test the energy threshold for the dosimetry systems, which can be generally an issue for those based on track detectors.

\section{The radiation fields at NPL}

Irradiations at NPL were performed using physically small cylindrical ${ }^{252} \mathrm{Cf}$ sources $(<2 \mathrm{~cm}$ high and $1 \mathrm{~cm}$ diameter).The dosemeters were attached to the front face of an ISO water-filled slab phantom. The midpoint of the front face was positioned at $75 \mathrm{~cm}$ from the centre of the source. Two sources were used; all irradiations but one with a source having an emission rate of $2.9 \times 10^{8} \mathrm{~s}^{-1}$. The $0.3-\mathrm{mSv}$ irradiation was performed with a lower output source of $3.4 \times 10^{7} \mathrm{~s}^{-1}$ to avoid timing problems. No irradiation took $>2.5 \mathrm{~h}$.

Source emission rates had been measured in the NPL manganese bath and the emission anisotropy using a long counter. Irradiations were performed in the low-scatter area, which has dimensions of $24 \times 18 \times 18 \mathrm{~m}^{3}$. The contribution from scattered neutrons could be neglected. Fluence-to-dose equivalent conversion coefficients were taken from ISO $8529-3^{(3)}$.

\section{The radiation fields at $P T B$}

Monoenergetic neutrons with the energy $(248 \pm 10$ $\mathrm{keV}$ ) were produced at the accelerator facility of the $\mathrm{PTB}^{(4)}$. Four dosemeters were irradiated with normally incident neutrons on an ISO water phantom (phantom-to-target distance: $75 \mathrm{~cm}$ ).

The neutron source facility of the PTB was used for the irradiation with the field of a bare ${ }^{252} \mathrm{Cf}$ source behind a shadow cone. The size of the concreteshielded irradiation room at PTB is $7 \mathrm{~m} \times 7 \mathrm{~m} \times 6.5$ $\mathrm{m}^{3}$, with the source in the centre. The neutron field behind a shadow cone is an isotropic field of wallscattered neutrons. The fluence and the spectral distribution of the scattered neutrons have been determined using the PTB Bonner-sphere spectrometer. $H_{\mathrm{p}}(10)$ was determined using the energy-dependent fluenceto-neutron personal dose equivalent conversion coefficients for isotropic incidence ${ }^{(5)}$.

\section{Information given to participantss}

The intercomparison was intended to be a blind test for the participants who provided their results without knowing the details of the irradiation performed (i.e. radiation fields, dose values, angle of incidence of the radiation, etc.). In neutron dosimetry, however, some information on the radiation field can be required, especially for albedo systems.

Therefore, the participants were asked to provide the results in two steps when different information was provided beforehand: 
Table 1. Radiation field information provided to the participants in the second step.

Irradiation conditions

Information provided

Bare ${ }^{252} \mathrm{Cf}$ source at $0^{\circ}, 45^{\circ}$

Bare radionuclide source

250-keV monoenergetic neutrons at $0^{\circ}$

$250 \mathrm{keV}$ monoenergetic neutrons

${ }^{252} \mathrm{Cf}\left(\mathrm{D}_{2} \mathrm{O}\right.$ moderated $)$ at $0^{\circ}$ and bare ${ }^{252} \mathrm{Cf}$ behind a

Radionuclide source with shadow cone significant moderated neutron fluence component

- First step: with little or no information on the radiation fields: 'Dosemeters were irradiated in groups with different neutron spectra: radionuclide sources, monoenergetic fields or workplace fields. Some of the fields contained significant contributions from slow and intermediate energy neutrons. No additional gamma component was added to the field over and above that associated with the neutron production. No information on dose, radiation quality, or the angle of the incident radiation will be given at this stage'.

- Second step: with information on the radiation fields as reported in Table 1, though it was up to the IMS to choose the proper calibration factor to be applied.

Participants were allowed to change their results between the first and second steps only according to their routine procedure, which had to be described and justified.

Some of the participants remarked, for some of their results, that the radiation field was not appropriate or that they were aware that their dosimetric procedure was not appropriate for certain radiation fields.

After the dose evaluation was provided by the IMSs, the reported dose values were compared with the reference doses given by the Irradiation Laboratories by calculating the response value $R$ :

$$
R=\frac{H_{\mathrm{p}}(10)_{\text {participant }}}{H_{\mathrm{p}}(10)_{\text {reference }}}
$$

\section{RESULTS}

The second-step results for all participants are shown in two graphs, with logarithmic $y$-axis, in Figure 1 according to the irradiation conditions and in Figure 2 for each dosimetry system.

The error bars in Figure 2 are not estimates of uncertainties but simply a measure of the spread of the results, the standard error of the mean in fact, for a particular irradiation field. They reveal some information about the data, e.g. they are relatively large for

the ${ }^{252} \mathrm{Cf}$ bare source behind a shadow cone, presumably because there were only two dosemeters irradiated and many of the responses are low. For the bare ${ }^{252} \mathrm{Cf}$ source fields the spreads are generally larger for the lowest dose rates where statistics are poor.

Most, but not all, participants performed acceptably well (within a factor of 2) for irradiation with a bare ${ }^{252} \mathrm{Cf}$ source at $0^{\circ}$, though most of the participants underestimate the reference value for an irradiation at $45^{\circ}$, causing concern for angle dependence of response. Results for $250-\mathrm{keV}$ monoenergetic neutrons vary considerably, not only reflecting the detection principle: monoenergetic fields are difficult for albedo systems and for etched track systems that have their fast neutron threshold close to this energy. The results with the simulated workplace fields, such as ${ }^{252} \mathrm{Cf}\left(\mathrm{D}_{2} \mathrm{O}\right)$, show quite good results for most of the systems, whilst results for the bare ${ }^{252} \mathrm{Cf}$ source behind a shadow cone mainly show an underestimation.

\section{DISCUSSION}

There is no 'internationally agreed' criterion for the performance of neutron dosemeters in individual monitoring. International Commission on Radiological Protection Publication $75^{(6)}$, when dealing with accuracy recommendations in individual dose assessment, states at $\$ 251$ that in workplace fields, where the energy spectrum and orientation of the radiation fields are usually not known, 'the overall uncertainty at the $95 \%$ confidence level in the estimation of effective dose around the relevant dose limit may well be a factor of 1.5 in either directions for photons and may be substantially greater for neutrons of uncertain energy and for electrons. Greater uncertainties are also inevitable at low levels of effective dose for all qualities of radiation'. The use of the factor 1.5 is mentioned in the ISO standard ${ }^{(7)}$ for photon dosimetry performance requirement and is used also for the well-known 'trumpet curve'. However, this would probably be too restrictive for neutron dosimetry. The OG decided to use a factor of 2 as a general criterion for the response, $R$, for all dose values. However, lines for a factor of 1.5 are also plotted in the graphs for comparative purposes.

The results need further analysis to report on the differences between the first- and second-step results as well as on-linearity and reproducibility for each dosimetry system. Such analysis will be provided in forthcoming publications.

\section{CONCLUSION}

The results of the IC2012n intercomparison are reported. Most, but not all, participants performed acceptably well (within a factor of 2) for all irradiation conditions. Good results were obtained in most 


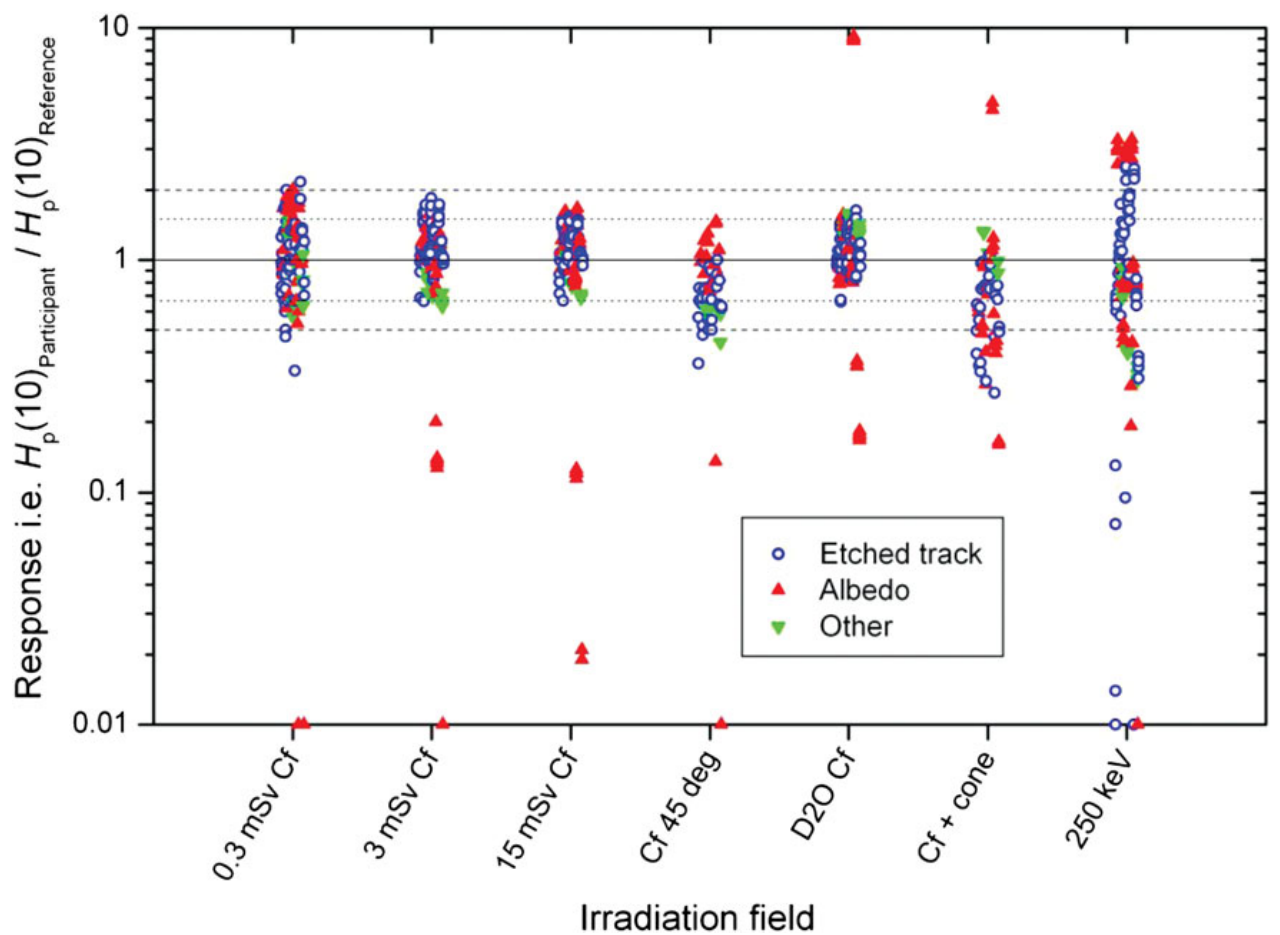

Figure 1. Overall results (second-step results) according to the irradiation conditions.

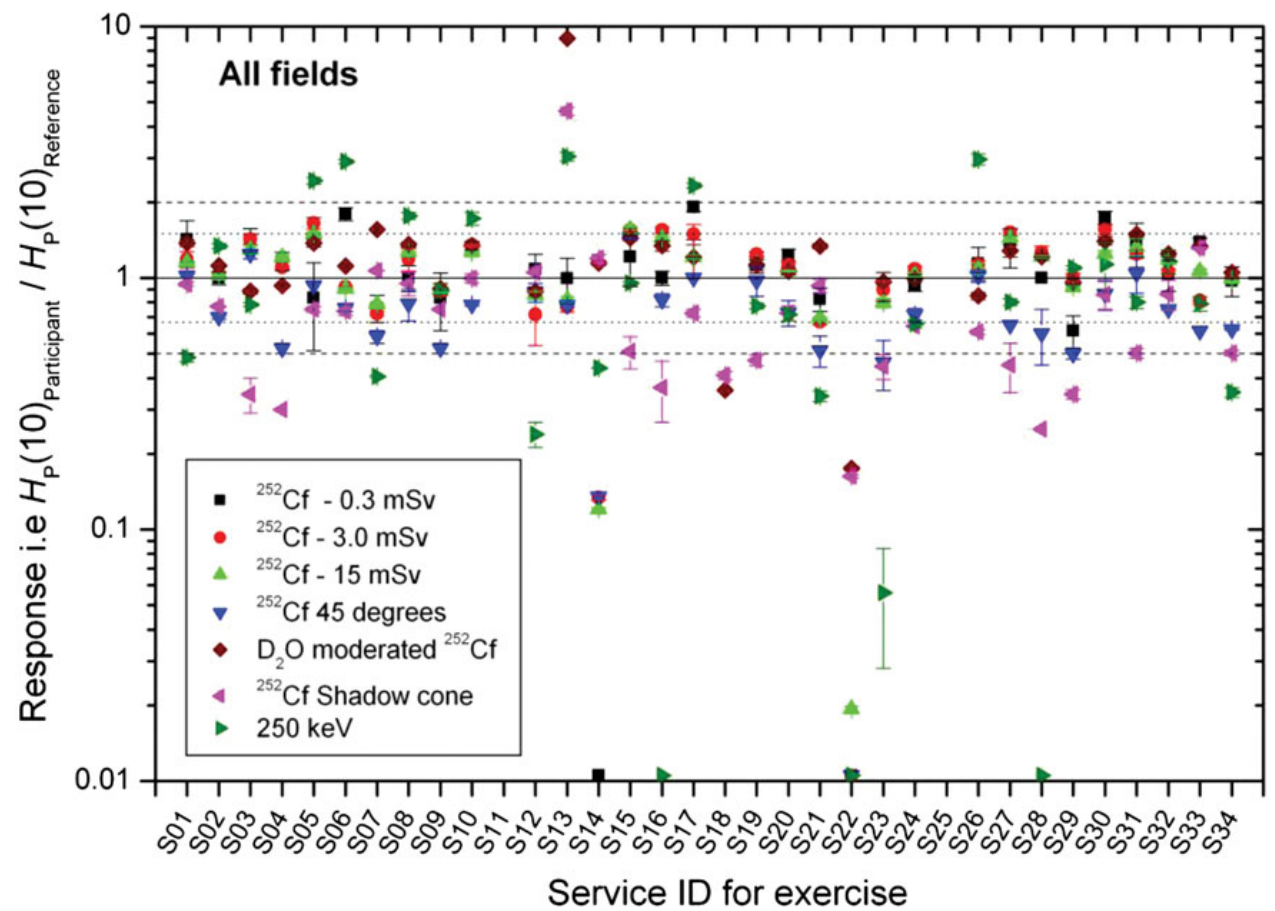

Figure 2. Overall results (second-step results) according to the dosimetry system (participant). 


\section{INTERCOMPARISON FOR NEUTRON PERSONAL DOSIMETRY}

radionuclide source radiation fields. A few participants reported poor results and some of them did not cover all irradiation conditions. Further analysis has to be performed to generate conclusions on the dosimetric techniques on which the dosemeters are based. The EURADOS IC21012 $\mathrm{n}$ is an important action in the field of regular performance tests in neutron dosimetry, for which intercomparisons at an international level have been performed only every $8-10$ y. A performance criterion for neutron dosimetry should be agreed internationally and the present intercomparison results can assist with this aim.

\section{REFERENCES}

1. European Commission. Technical recommendations for monitoring individuals occupationally exposed to external radiation. Directorate-General for Energy and Transport. RP 160. EC (2009).

2. Grimbergen, T. W. M., Figel, M., Romero, A. M., Stadtmann, H. and McWhan, A. F. EURADOS self- sustained programme of intercomparisons for individual monitoring services. Radiat. Prot. Dosim. 144(1-4), 266-274 (2011).

3. International Organization for Standardisation. Reference neutron irradiations - Part 3: calibration of area and personal dosimeters and determination of their response as a function of neutron energy and angle of incidence. ISO Standard 8529-3. ISO (1998).

4. Nolte, R. et al. Quasi-monoenergetic neutron reference fields in the energy range from thermal to $200 \mathrm{MeV}$. Radiat. Prot. Dosim. 110(1-4), 97-102 (2004).

5. Kluge, H. Irradiation facility with radioactive reference neutron sources: basic principles. Report PTB-N-34. Physikalisch-Technische Bundesanstalt (1998). ISBN 389701-192-1.

6. International Commission on Radiological Protection. General principles for the radiation protection of workers. ICRP publication 75. Ann. ICRP 27(1). Pergamon (1997).

7. International Organization for Standardisation. Radiation protection-criteria and performance limits for the periodic evaluation of processors of personal dosemeters for $X$ and gamma radiation. ISO 14146. ISO (2000). 